\title{
$h$ TRANSFORM OF ONE-DIMENSIONAL GENERALIZED DIFFUSION OPERATORS
}

\author{
Tomoko TAKEMURA and Matsuyo TOMISAKI
}

(Received 7 February 2011 and revised 31 July 2011)

\begin{abstract}
We are concerned with two types of $h$ transform of one-dimensional generalized diffusion operators treated by Maeno (2006) and by Tomisaki (2007). We show that these two types of $h$ transform are in inverse relation to each other in some sense. Further, we show that a recurrent one-dimensional generalized diffusion operator cannot be represented as an $h$ transform of another one-dimensional generalized diffusion operator different from the original one. We also consider a spectral representation of elementary solutions corresponding to $h$ transformed one-dimensional generalized diffusion operators.
\end{abstract}

\section{Introduction}

Let $\mathcal{G}_{s, m, k}$ be a one-dimensional generalized diffusion operator on an interval $I$ with scale function $s$, speed measure $m$, and killing measure $k$ (ODGDO with $(s, m, k)$ for brevity). Let $\mathcal{M}_{s, m}$ be the set of all positive functions $h$ satisfying $\mathcal{G}_{s, m, 0} h \leq 0$, where $\mathcal{G}_{s, m, 0}$ is an ODGDO with $(s, m, 0)$ and 0 denotes the null killing measure. For $\beta \geq 0$, let $\mathcal{H}_{s, m, k, \beta}$ be the set of all positive functions satisfying $\mathcal{G}_{s, m, k} h=\beta h$. In this paper, we are concerned with two types of $h$ transform based on $h \in \mathcal{M}_{s, m}$ and $h \in \mathcal{H}_{s, m, k, \beta}$.

It is well known that the generator of a one-dimensional generalized diffusion process (ODGDP) is represented as an ODGDO. In Section 4.3 of [3], Itô and McKean used a method to derive such a general form $\mathcal{G}_{s, m, k}$ for the generator of ODGDP. Their method is an $h$ transform based on the probability which the sample paths hit one of the end points of the state interval. Following their idea, Maeno [4] considered $h$ transforms of ODGDPs based on $h \in \mathcal{M}_{s, m}$ and studied some properties corresponding to $h$ transformed ODGDP. On the other hand, Tomisaki [8] considered $h$ transforms of ODGDOs based on $h \in \mathcal{H}_{s, m, k, \beta}$ and obtained some results for $h$ transformed ODGDOs. As we will see in Proposition 1.1 below, the sets $\mathcal{M}_{s, m}$ and $\mathcal{H}_{s, m, k, \beta}$ are usually disjoint to each other. Therefore the results of [4] and [8] are not necessarily derived from each other.

PROPOSITION 1.1.

(i) The set $\mathcal{H}_{s, m, 0,0}$ coincides with the set $\left\{h \in \mathcal{M}_{s, m} ; D_{s} h(x)\right.$ is a constant function on $\left.I\right\}$.

(ii) If $k$ is not a null measure or $\beta>0$, then $\mathcal{M}_{s, m} \cap \mathcal{H}_{s, m, k, \beta}=\emptyset$.

2000 Mathematics Subject Classification: Primary 60J60, 60G20.

Keywords: generalized diffusion process; harmonic transformation; recurrence.

(c) 2012 Faculty of Mathematics, Kyushu University 
Here $D_{s} h$ is the right derivative of $h$ with respect to the scale function $s$. We show this proposition in Section 3.

Let $p(t, x, y)$ be the elementary solution of the equation

$$
\frac{\partial}{\partial t} p(t, x, y)=\mathcal{G}_{s, m, k} p(t, x, y), \quad t>0, x, y \in I,
$$

in the sense of McKean [5], where $\mathcal{G}_{s, m, k}$ is applied to $x$ or $y$.

Let $p^{o}(t, x, y)$ be the elementary solution of equation (1.1) with $\mathcal{G}_{s, m, k}$ replaced by $\mathcal{G}_{s, m, 0}$. For $h \in \mathcal{M}_{s, m}$, we set

$$
\begin{aligned}
p_{h}^{o}(t, x, y) & =p^{o}(t, x, y) / h(x) h(y), \quad t>0, x, y \in I, \\
s_{h}(x) & =\int_{\left(c_{o}, x\right]} h(y)^{-2} d s(y), \\
m_{h}(x) & =\int_{\left(c_{o}, x\right]} h(y)^{2} d m(y), \\
k_{h}(x) & =-\int_{\left(c_{o}, x\right]} h(y) d D_{s} h(y),
\end{aligned}
$$

where $c_{o}$ is a point of $I$ fixed arbitrarily. Maeno [4] showed that $p_{h}^{o}(t, x, y)$ is the elementary solution of equation (1.1) with $\mathcal{G}_{s_{h}, m_{h}, k_{h}}$ in place of $\mathcal{G}_{s, m, k}$. Further, Maeno studied the asymptotic behavior near the boundaries of $I$ for sample paths of ODGDP with generator $\mathcal{G}_{s_{h}, m_{h}, k_{h}}$, and gave a precise classification of the states of the boundaries by means of $s, m$ and $h$. We call $\mathcal{G}_{s_{h}, m_{h}, k_{h}}$ the $h$ transform of $\mathcal{G}_{s, m, 0}$ with $h \in \mathcal{M}_{s, m}$.

We next turn to an $h$ transform treated in [8]. Let $p(t, x, y)$ be the elementary solution of equation (1.1). For $h \in \mathcal{H}_{s, m, k, \beta}$ set

$$
p_{h}^{*}(t, x, y)=e^{-\beta t} p(t, x, y) / h(x) h(y), \quad t>0, x, y \in I .
$$

Then $p_{h}^{*}(t, x, y)$ is the elementary solution of equation (1.1) with $\mathcal{G}_{s_{h}, m_{h}, 0}$ in place of $\mathcal{G}_{s, m, k}$, where $s_{h}$ and $m_{h}$ are given by (1.3) and (1.4), respectively. We note that Takemura [7] studied some asymptotic properties of sample paths near the boundaries of $I$ for ODGDP with generator $\mathcal{G}_{s_{h}, m_{h}, 0}$, and obtained a precise classification of the states of the boundaries by means of $s, m, k$ and $h$. We call $\mathcal{G}_{s_{h}, m_{h}, 0}$ the $h$ transform of $\mathcal{G}_{s, m, k}$ with $h \in \mathcal{H}_{s, m, k, \beta}$.

For $h \in \mathcal{M}_{s, m}$ (respectively $h \in \mathcal{H}_{s, m, k, \beta}$ ), we set $H_{h}^{o} \mathcal{G}_{s, m, 0} u=h^{-1} \mathcal{G}_{s, m, 0}(h u)$ (respectively $\left.H_{h}^{*} \mathcal{G}_{s, m, k+\beta m} u=h^{-1} \mathcal{G}_{s, m, k+\beta m}(h u)\right)$ for $u$ satisfying $h u \in D\left(\mathcal{G}_{s, m, 0}\right)$ (respectively $h u \in D\left(\mathcal{G}_{s, m, k+\beta m}\right)$ ). In the case that $\mathcal{G}_{s, m, 0}$ and $\mathcal{G}_{s, m, k}$ are differential operators of second order, it is easy to see that $H_{h}^{o} \mathcal{G}_{s, m, 0}$ and $H_{h}^{*} \mathcal{G}_{s, m, k+\beta m}$ coincide with $\mathcal{G}_{s_{h}, m_{h}, k_{h}}$ and $\mathcal{G}_{s_{h}, m_{h}, 0}$, respectively. We show that this is also true for ODGDOs.

THEOREM 1.2. Let u be a measurable function on I.

(i) Let $h \in \mathcal{M}_{s, m}$. Then $u$ belongs to $D\left(\mathcal{G}_{s_{h}, m_{h}, k_{h}}\right)$ if and only if $h u$ belongs to $D\left(\mathcal{G}_{s, m, 0}\right)$.

Further, $\mathcal{G}_{s_{h}, m_{h}, k_{h}} u=H_{h}^{o} \mathcal{G}_{s, m, 0} u$ holds true for $u \in D\left(\mathcal{G}_{s_{h}, m_{h}, k_{h}}\right)$.

(ii) Let $h \in \mathcal{H}_{s, m, k, \beta}$. Then $u$ belongs to $D\left(\mathcal{G}_{s_{h}, m_{h}, 0}\right)$ if and only if hu belongs to $D\left(\mathcal{G}_{s, m, k}\right)$.

Further, $\mathcal{G}_{s_{h}, m_{h}, 0} u=H_{h}^{*} \mathcal{G}_{s, m, k+\beta m} u$ holds true for $u \in D\left(\mathcal{G}_{s_{h}, m_{h}, 0}\right)$.

COROLlaRY 1.3.

(i) Let $h \in \mathcal{H}_{s, m, 0,0}$. Then $H_{h}^{o} \mathcal{G}_{s, m, 0}=H_{h}^{*} \mathcal{G}_{s, m, 0}=\mathcal{G}_{s_{h}, m_{h}, 0}$. 
(ii) Let $h \in \mathcal{M}_{s, m}$. Then $H_{h}^{o} \mathcal{G}_{s, m, 0}=\mathcal{G}_{s, m, 0}$ if and only if $h$ is a positive constant function.

(iii) Let $h \in \mathcal{H}_{s, m, k, \beta}$. Then $H_{h}^{*} \mathcal{G}_{s, m, k+\beta m}=\mathcal{G}_{s, m, k+\beta m}$ if and only if $k$ is the null measure, $\beta=0$, and $h$ is a positive constant function.

We next show that $H_{h}^{o}$ and $H_{h}^{*}$ are in inverse relation to each other in some sense.

THEOREM 1.4.

(i) Let $h \in \mathcal{M}_{s, m}$. Then $h^{-1}$ belongs to $\mathcal{H}_{s_{h}, m_{h}, k_{h}, 0}$ and $H_{h^{-1}}^{*} H_{h}^{o} \mathcal{G}_{s, m, 0}=\mathcal{G}_{s, m, 0}$.

(ii) Let $h \in \mathcal{H}_{s, m, k, \beta}$. Then $h^{-1}$ belongs to $\mathcal{M}_{s_{h}, m_{h}}$ and $H_{h^{-1}}^{o} H_{h}^{*} \mathcal{G}_{s, m, k+\beta m}=\mathcal{G}_{s, m, k+\beta m}$.

We should note that Theorem 1.4 does not necessarily ensure one-to-one correspondence of an $h$ transform. Indeed, some examples are given in Section 6, which show that an $h$ transform of an ODGDO is not a one-to-one correspondence.

Now Theorem 1.2 implies that an ODGDO is represented as an $h$ transform of another ODGDO, but it does not always imply that an ODGDO is represented as an $h$ transform of another ODGDO different from the original one. As we will see in the following theorem, this problem is related to a global property of ODGDOs. We denote by $\Phi$ the set of all ODGDOs on $I$. Let $\Phi^{R}$ be the set of all recurrent ODGDOs on $I$, that is,

$$
\Phi^{R}=\left\{\mathcal{G}_{s, m, k} \in \Phi: s\left(l_{1}\right)=-\infty, s\left(l_{2}\right)=\infty \text {, and } k \text { is the null measure }\right\} .
$$

We set $\Phi^{T}=\Phi \backslash \Phi^{R}$, which is the set of all transient ODGDOs on $I$.

THEOREM 1.5 .

(i) Let $\mathcal{G}_{s, m, 0} \in \Phi^{R}$. If $H_{h}^{o} \mathcal{G}_{s, m, 0} \in \Phi^{R}$ for some $h \in \mathcal{M}_{s, m}$, then $h$ is a positive constant function. If $H_{h}^{*} \mathcal{G}_{s, m, \beta m} \in \Phi^{R}$ for some $h \in \mathcal{H}_{s, m, 0, \beta}$, then $h$ is a positive constant function and $\beta=0$.

(ii) Let $\mathcal{G}_{s, m, 0} \in \Phi^{R}$. If $H_{h}^{o} \mathcal{G}_{s, m, 0} \in \Phi^{T}$ for some $h \in \mathcal{M}_{s, m}$, then $h$ is not a positive constant function. If $H_{h}^{*} \mathcal{G}_{s, m, \beta m} \in \Phi^{T}$ for some $h \in \mathcal{H}_{s, m, 0, \beta}$, then $h$ is not a positive constant function or $\beta>0$. Conversely, if $h \in \mathcal{M}_{s, m}$ and $h$ is not a positive constant function, then $H_{h}^{o} \mathcal{G}_{s, m, 0} \in \Phi^{T}$. If $h \in \mathcal{H}_{s, m, 0, \beta}$ and either $h$ is not a positive constant function or $\beta>0$, then $H_{h}^{*} \mathcal{G}_{s, m, \beta m} \in \Phi^{T}$.

(iii) If $\mathcal{G}_{s, m, 0} \in \Phi^{T}$, then $H_{h}^{o} \mathcal{G}_{s, m, 0} \in \Phi^{T}$ for any $h \in \mathcal{M}_{s, m}$. If $\mathcal{G}_{s, m, k} \in \Phi^{T}$, then $H_{h}^{*} \mathcal{G}_{s, m, k+\beta m} \in \Phi^{T}$ for any $h \in \mathcal{H}_{s, m, k, \beta}$.

We will show this theorem in Section 4.

We finally consider spectral representations of elementary solutions. We assume that $\operatorname{supp}[m]=I$ and $l_{1}$ is not $(s, m, k)$-natural. Further, we assume that $p(t, x, y)$ is represented as

$$
p(t, x, y)=\int_{[0, \infty)} e^{-\lambda t} \psi(x, \lambda) \psi(y, \lambda) d \sigma(\lambda), \quad t>0, x, y \in I,
$$

where $d \sigma(\lambda)$ is a Borel measure on $[0, \infty)$ such that $\sigma(\{0\})=0$ if $l_{1}$ is $(s, m, k)$-regular or -exit. Further, $\psi(x, \lambda), x \in I, \lambda \geq 0$, is the solution of the integral equation (5.1) or (5.2) below. Let $h \in \mathcal{H}_{s, m, k, \beta}$ and $p_{h}^{*}(t, x, y)$ be the elementary solution of equation (1.1) with $\mathcal{G}_{s_{h}, m_{h}, 0}$ in place of $\mathcal{G}_{s, m, k}$, which is given by (1.6). By means of (1.6) and (1.7), we suppose $p_{h}^{*}(t, x, y)$ is represented as

$$
p_{h}^{*}(t, x, y)=\int_{[\beta, \infty)} e^{-\lambda t} \psi_{h}^{*}(x, \lambda) \psi_{h}^{*}(y, \lambda) d \sigma_{h}^{*}(\lambda), \quad t>0, x, y \in I,
$$


and $\psi_{h}^{*}(x, \lambda)$ and $d \sigma_{h}^{*}(\lambda)$ are represented as

$$
\psi_{h}^{*}(x, \lambda)=C_{o} \psi(x, \lambda-\beta) / h(x), \quad d \sigma_{h}^{*}(\lambda)=C_{o}^{-2} d_{\lambda} \sigma(\lambda-\beta),
$$

for $\lambda \geq \beta$, where $C_{o}$ is a positive constant function. In Section 5 we show that $\psi_{h}^{*}(x, \lambda)$ satisfies (5.1) or (5.2) with $(s, m, k)$ replaced by $\left(s_{h}, m_{h}, 0\right)$. Further, we will find there that $C_{o}$ only depends on the behavior of $s, m, k$ and $h$ near the boundary $l_{1}$, which leads us to an interesting behavior of Lévy measure density of inverse local time corresponding to a diffusion process on $I$ with the scale function $s_{h}$ and the speed measure $m_{h}$. We will discuss this in another paper.

The organization of this paper is as follows. In Section 2 we give the precise definitions of ODGDO $\mathcal{G}_{s, m, k}$, the domain $D\left(\mathcal{G}_{s, m, k}\right)$ and the corresponding items. In Section 3 we give the precise definitions of $\mathcal{M}_{s, m}$ and $\mathcal{H}_{s, m, k, \beta}$, and prove Proposition 1.1 and Theorems 1.2 and 1.4. In Section 4 we prove Theorem 1.5. In Section 5 we show that (1.8) holds true for $\psi_{h}^{*}(x, \lambda)$ and $d \sigma_{h}^{*}(\lambda)$ given by (1.9) with a suitable constant $C_{o}$. It is easy to see that if $H_{h_{1}}^{o} \mathcal{G}_{s, m, 0}=H_{h_{2}}^{o} \mathcal{G}_{s, m, 0}$ (respectively $H_{h_{1}}^{*} \mathcal{G}_{s, m, k+\beta m}=H_{h_{2}}^{*} \mathcal{G}_{s, m, k+\beta m}$ ) for some $h_{1}, h_{2} \in \mathcal{M}_{s, m}$ (respectively $h_{1}, h_{2} \in \mathcal{H}_{s, m, k, \beta}$ ), then there is a positive constant $K$ such that $h_{1}=K h_{2}$. However, it is not necessarily true that if $H_{h_{1}}^{o} \mathcal{G}_{s_{1}, m_{1}, 0}=H_{h_{2}}^{o} \mathcal{G}_{s_{2}, m_{2}, 0}$ (respectively $H_{h_{1}}^{*} \mathcal{G}_{s_{1}, m_{1}, k_{1}+\beta_{1} m_{1}}=H_{h_{2}}^{*} \mathcal{G}_{s_{2}, m_{2}, k_{2}+\beta_{2} m_{2}}$ ) for some $h_{i} \in \mathcal{M}_{s_{i}, m_{i}}$ (respectively $\left.h_{i} \in \mathcal{H}_{s_{i}, m_{i}, k_{i}, \beta_{i}}\right)(i=1,2)$, then $h_{1}=K h_{2}$ for some positive constant $K$ and $\mathcal{G}_{s_{1}, m_{1}, 0}=$ $\mathcal{G}_{s_{2}, m_{2}, 0}$ (respectively $\mathcal{G}_{s_{1}, m_{1}, k_{1}+\beta_{1} m_{1}}=\mathcal{G}_{s_{2}, m_{2}, k_{2}+\beta_{2} m_{2}}$ ). We give such typical examples in Section 6.

\section{Preliminaries}

In this section we give the precise definitions of ODGDO $\mathcal{G}_{s, m, k}$, the domain $D\left(\mathcal{G}_{s, m, k}\right)$ and the corresponding items.

Let $s$ be a continuous increasing function on an open interval $I=\left(l_{1}, l_{2}\right)$, where $-\infty \leq$ $l_{1}<l_{2} \leq \infty$, let $m$ be a right-continuous nondecreasing function on $I$ and let $k$ be a rightcontinuous nondecreasing function on $I$. We sometimes use the same symbols $s, m$ and $k$ for the induced measures $d s(x), d m(x)$ and $d k(x)$, respectively. For a function $u$ on $I$, we set $u\left(l_{i}\right)=\lim _{x \rightarrow l_{i}, x \in I} u(x)$ if the limit exists, for $i=1,2$. We set

$$
I_{*}(\mu)=\left\{x \in I ; \mu\left(x_{1}\right)<\mu\left(x_{2}\right) \text { for } \ell_{1}<{ }^{\forall} x_{1}<x<{ }^{\forall} x_{2}<\ell_{2}\right\},
$$

for a nondecreasing right-continuous function $\mu$ on $I . I_{*}(\mu)$ is the same as the support of the measure induced by $\mu$. We assume $I_{*}(m) \neq \emptyset$ and $I_{*}(k) \subset I_{*}(m)$ throughout this paper. Further, we set

$$
I_{* *}(m)=I_{*}(m) \cup\left\{x ; x=\ell_{i} \text { with }\left|m\left(\ell_{i}\right)\right|+\left|s\left(\ell_{i}\right)\right|+\left|k\left(\ell_{i}\right)\right|<\infty, i=1,2\right\} .
$$

Let us fix a point $c_{o} \in I_{*}(m)$ arbitrarily and set

$$
J_{\mu, v}(x)=\int_{\left(c_{o}, x\right]} d \mu(y) \int_{\left(c_{o}, y\right]} d v(z)
$$


for $x \in I$, where $\mu$ and $v$ are Borel measures on $I$, and the integral $\int_{(a, b]}$ is read as $-\int_{(b, a]}$ if $a>b$. Following [1], we say the boundary $l_{i}$ is

$$
\begin{array}{ll}
(s, m, k) \text {-regular } & \text { if } J_{s, m+k}\left(l_{i}\right)<\infty \text { and } J_{m+k, s}\left(l_{i}\right)<\infty, \\
(s, m, k) \text {-exit } & \text { if } J_{s, m+k}\left(l_{i}\right)<\infty \text { and } J_{m+k, s}\left(l_{i}\right)=\infty, \\
(s, m, k) \text {-entrance } & \text { if } J_{s, m+k}\left(l_{i}\right)=\infty \text { and } J_{m+k, s}\left(l_{i}\right)<\infty, \\
(s, m, k) \text {-natural } & \text { if } J_{s, m+k}\left(l_{i}\right)=\infty \text { and } J_{m+k, s}\left(l_{i}\right)=\infty .
\end{array}
$$

Let $D\left(\mathcal{G}_{s, m, k}\right)$ be the space of all functions $u \in L^{2}(I, m)$ which have a continuous version $u$ (we use the same symbol) satisfying the following conditions:

(i) there exist two constants $A, B$ and a function $f_{u} \in L^{2}(I, m)$ such that

$$
\begin{aligned}
u(x)= & A+B s(x)+\int_{\left(c_{o}, x\right]}\{s(x)-s(y)\} f_{u}(y) d m(y) \\
& +\int_{\left(c_{o}, x\right]}\{s(x)-s(y)\} u(y) d k(y), \quad x \in I
\end{aligned}
$$

(ii) $\quad$ if $l_{i}$ is $(s, m, k)$-regular, then $u\left(l_{i}\right)=0$ for each $i=1,2$.

By virtue of (2.2), $f_{u}$ is uniquely determined as a function of $L^{2}(I, m)$ if it exists. The operator $\mathcal{G}_{s, m, k}$ from $D\left(\mathcal{G}_{s, m, k}\right)$ into $L^{2}(I, m)$ is defined by $\mathcal{G}_{s, m, k} u=f_{u}$, and it is called the one-dimensional generalized diffusion operator with the scale function $s$, the speed measure $m$, and the killing measure $k$ (ODGDO with $(s, m, k)$ ). The above condition (ii) implies that the absorbing boundary condition is posed at the regular boundary.

It is easy to see that $\mathcal{G}_{s_{1}, m_{1}, k_{1}}$ coincides with $\mathcal{G}_{s_{2}, m_{2}, k_{2}}$ if and only if there are a positive constant $K$ and constants $K_{i}, i=1,2,3$, such that $s_{1}=K s_{2}+K_{1}, m_{1}=K^{-1} m_{2}+K_{2}$, and $k_{1}=K^{-1} k_{2}+K_{3}$ (see [3]).

We note that $l_{i}$ is $(s, m, k+\beta m)$-regular, -exit, -entrance, or -natural according to if $l_{i}$ is $(s, m, k)$-regular, exit, entrance, or natural, for $i=1,2$ and $\beta \geq 0$. Combining this fact with (2.2), we immediately obtain the following:

$$
\begin{aligned}
D\left(\mathcal{G}_{s, m, k}\right) & =D\left(\mathcal{G}_{s, m, k+\beta m}\right), \\
\mathcal{G}_{s, m, k} u-\beta u & =\mathcal{G}_{s, m, k+\beta m} u \quad \text { for } u \in D\left(\mathcal{G}_{s, m, k}\right),
\end{aligned}
$$

where $\beta \geq 0$.

In the following, for a measurable function $u$ on $I, D_{s} u(x)$ stands for the right derivative with respect to $s(x)$, that is, $D_{s} u(x)=\lim _{\varepsilon \downarrow 0}\{u(x+\varepsilon)-u(x)\} /\{s(x+\varepsilon)-s(x)\}$, provided it exists. It is obvious that $u \in D\left(\mathcal{G}_{s, m, k}\right)$ has the right derivative $D_{s} u$ and it satisfies

$$
D_{s} u(y)-D_{s} u(x)=\int_{(x, y]} \mathcal{G}_{s, m, k} u(z) d m(z)+\int_{(x, y]} u(z) d k(z), \quad x, y \in I .
$$

So we sometimes use the symbol $\mathcal{G}_{s, m, k} u=\left(d D_{s} u-u d k\right) / d m$.

Following McKean [5] (see also Section 4.11 of [3]), we can define the elementary solution $p(t, x, y)$ of equation (1.1). It is known that $p(t, x, y)$ satisfies the following 
properties:

$$
\begin{aligned}
& p(t, x, y)=p(t, y, x)>0, \quad t>0, x, y \in I \\
& p(t, x, y) \text { is continuous on }(0, \infty) \times I \times I ; \\
& p(s+t, x, y)=\int_{I} p(s, x, z) p(t, z, y) d m(z), \quad s, t>0, x, y \in I ; \\
& p\left(t, l_{i}, y\right)=0, \quad t>0, y \in I, \quad \text { if } l_{i} \text { is not entrance; } \\
& D_{s} p\left(t, l_{i}, y\right)=0, \quad t>0, y \in I, \quad \text { if } l_{i} \text { is entrance, }
\end{aligned}
$$

where $D_{s} p(t, x, y)=\lim _{\varepsilon \downarrow 0}\{p(t, x+\varepsilon, y)-p(t, x, y)\} /\{s(x+\varepsilon)-s(x)\}$. It is also known that there exists a ODGDP $\mathbb{D}_{s, m, k}=\left[X(t): t \geq 0, P_{x}: x \in I_{* *}(m)\right]$ such that

$$
P_{x}(X(t) \in E)=\int_{E} p(t, x, y) d m(y), \quad t>0, x \in I_{* *}(m), E \in \mathcal{B}(I),
$$

where $\mathcal{B}(I)$ stands for the set of all Borel sets of $I$. For this reason, $p(t, x, y)$ is sometimes called the transition probability density with respect to $m$.

For $\alpha \geq 0$ and $i=1,2$, let $g_{i}(\cdot, \alpha)$ be a function on $I$ satisfying the following properties (2.5)-(2.9):

$$
\begin{aligned}
& g_{i}(x, \alpha) \text { is positive and continuous in } x \text {; } \\
& g_{1}(x, \alpha) \text { is nondecreasing in } x ; \\
& g_{2}(x, \alpha) \text { is nonincreasing in } x ; \\
& g_{i}\left(l_{i}, \alpha\right)=0 \text { if }\left|s\left(l_{i}\right)\right|<\infty \\
& \begin{aligned}
g_{i}(x, \alpha)= & g_{i}\left(c_{o}, \alpha\right)+D_{s} g_{i}\left(c_{o}, \alpha\right)\left\{s(x)-s\left(c_{o}\right)\right\} \\
& +\int_{\left(c_{o}, x\right]}\{s(x)-s(y)\} g_{i}(y, \alpha)\{\alpha d m(y)+d k(y)\}, \quad x \in I .
\end{aligned}
\end{aligned}
$$

Here, $\quad D_{s} g_{i}(x, \alpha)=\lim _{\varepsilon \downarrow 0}\left\{g_{i}(x+\varepsilon, \alpha)-g_{i}(x, \alpha)\right\} /\{s(x+\varepsilon)-s(x)\}, i=1,2 . \quad$ It $\quad$ is known that there exist functions $g_{i}(\cdot, \alpha), i=1,2$, satisfying the properties (2.5)-(2.9) (see Section 4.6 of [3]). We summarize some properties of $g_{i}(\cdot, \alpha), i=1,2$, which we need later.

\section{PROPOSITION 2.1.}

(i) [3] Assume that $k$ is not a null measure or $\alpha>0$. Then it holds true that:

$$
\begin{aligned}
& g_{i}\left(l_{i}, \alpha\right) \begin{cases}\in(0, \infty) & \text { if } l_{i} \text { is }(s, m, k) \text {-entrance, } \\
=0 & \text { if } l_{i} \text { is not }(s, m, k) \text {-entrance } ;\end{cases} \\
& g_{j}\left(l_{i}, \alpha\right) \begin{cases}\in(0, \infty) & \text { if } l_{i} \text { is }(s, m, k) \text {-regular or -exit, } \\
=\infty & \text { if } l_{i} \text { is }(s, m, k) \text {-entrance or -natural; }\end{cases}
\end{aligned}
$$




$$
\begin{aligned}
& \left|D_{s} g_{i}\left(l_{i}, \alpha\right)\right| \begin{cases}\in(0, \infty) & \text { if } l_{i} \text { is }(s, m, k) \text {-regular or -exit, } \\
=0 & \text { if } l_{i} \text { is }(s, m, k) \text {-entrance } \text { or -natural; }\end{cases} \\
& \left|D_{s} g_{j}\left(l_{i}, \alpha\right)\right| \begin{cases}\in(0, \infty) & \text { if } l_{i} \text { is }(s, m, k) \text {-regular or-entrance } \\
=\infty & \text { if } l_{i} \text { is }(s, m, k) \text {-exit or-natural }\end{cases} \\
& \lim _{x \rightarrow l_{i}} g_{i}(x, \alpha) D_{s} g_{j}(x, \alpha)=0 \quad \text { if } l_{i} \text { is }(s, m, k) \text {-exit, } \\
& \lim _{x \rightarrow l_{i}} g_{j}(x, \alpha) D_{s} g_{i}(x, \alpha)=0 \quad \text { if } l_{i} \text { is }(s, m, k) \text {-entrance }
\end{aligned}
$$

where $i, j=1,2$ and $i \neq j$.

(ii) Assume that $k$ is the null measure and $\alpha=0$. Then $g_{i}(x, 0), i=1,2$ are represented as follows:

$$
\begin{aligned}
& g_{1}(x, 0)= \begin{cases}C_{1} & \text { if } s\left(l_{1}\right)=-\infty \\
C_{1}\left\{s(x)-s\left(l_{1}\right)\right\} & \text { if } s\left(l_{1}\right)>-\infty\end{cases} \\
& g_{2}(x, 0)= \begin{cases}C_{2} & \text { if } s\left(l_{2}\right)=\infty, \\
C_{2}\left\{s\left(l_{2}\right)-s(x)\right\} & \text { if } s\left(l_{2}\right)<\infty\end{cases}
\end{aligned}
$$

where $C_{1}$ and $C_{2}$ are positive constants.

Statement (i) is shown in Section 4.6 of [3] and statement (ii) follows from (2.5)-(2.8) hence we omit the proof.

We set $W(\alpha)=D_{s} g_{1}(x, \alpha) g_{2}(x, \alpha)-g_{1}(x, \alpha) D_{s} g_{2}(x, \alpha)$. Note that $W(\alpha)$ is a positive number independent of $x \in I$. We put

$$
G(\alpha, x, y)=G(\alpha, y, x)=W(\alpha)^{-1} g_{1}(x, \alpha) g_{2}(y, \alpha),
$$

for $\alpha>0, x, y \in I, x \leq y$. We call $G(\alpha, x, y)$ the $\alpha$-Green function corresponding to the ODGDO $\mathcal{G}_{s, m, k}$. It is also known that

$$
G(\alpha, x, y)=\int_{0}^{\infty} e^{-\alpha t} p(t, x, y) d t, \quad \alpha>0, x, y \in I_{* *}(m) .
$$

It is easy to see that, if $k \neq 0$, then there exists $G(0, x, y)$ which is given by

$$
G(0, x, y)=G(0, y, x)=W^{-1} g_{1}(x) g_{2}(y), \quad x, y \in I, x \leq y,
$$

where $g_{i}(x)=g_{i}(x, 0), i=1,2$, and $W=D_{s} g_{1}(x) g_{2}(x)-g_{1}(x) D_{s} g_{2}(x)$, which is a positive constant independent of $x \in I$. It follows from Proposition 2.1 that, in the case $k=0$, there exists $G(0, x, y) \in(0, \infty)$ if and only if $\left|s\left(l_{i}\right)\right|<\infty$ for $i=1$ or 2 .

We denote by $G_{\alpha}(\alpha>0)$ the Green operator corresponding to $\mathcal{G}_{s, m, k}$ :

$$
G_{\alpha} f(x)=\int_{I} G(\alpha, x, y) f(y) d m(y), \quad f \in L^{2}(I, m) .
$$

It is well known that

$$
\begin{aligned}
& G_{\alpha}\left(L^{2}(I, m)\right)=D\left(\mathcal{G}_{s, m, k}\right), \\
& G_{\alpha}\left(\alpha-\mathcal{G}_{s, m, k}\right) u=u, \quad u \in D\left(\mathcal{G}_{s, m, k}\right), \\
& \left(\alpha-\mathcal{G}_{s, m, k}\right) G_{\alpha} f=f, \quad f \in L^{2}(I, m)
\end{aligned}
$$

(see [2] and [3]). 


\section{3. $h$ transform of ODGDOs}

In this section, we give the precise definitions of $\mathcal{M}_{s, m}$ and $\mathcal{H}_{s, m, k, \beta}$, and prove Proposition 1.1 and Theorems 1.2 and 1.4. We use the same notations as in the preceding sections.

\section{1. $h$ transform of $\mathcal{G}_{s, m, 0}$ with $h \in \mathcal{M}_{s, m}$}

Let $\mathcal{M}_{s, m}$ be the set of all positive continuous functions $h$ on $I$ such that $h$ has the right derivative $D_{s} h, D_{s} h$ is right continuous and nonincreasing, and the set $\left\{x \in I ; D_{s} h\left(x_{1}\right)>\right.$ $D_{s} h\left(x_{2}\right)$ for $\left.l_{1}<\forall x_{1}<x<\forall x_{2}<l_{2}\right\}$ is included in $I_{*}(m)$. For $h \in \mathcal{M}_{s, m}$, we consider $s_{h}$, $m_{h}$ and $k_{h}$ given by (1.3), (1.4) and (1.5), respectively. Note that $I_{*}(m)=I_{*}\left(m_{h}\right)$ and $I_{*}\left(k_{h}\right) \subset I_{*}\left(m_{h}\right)$ for $h \in \mathcal{M}_{s, m}$ (see [4]). Let $p^{o}(t, x, y)$ be the elementary solution of (1.1) with $\mathcal{G}_{s, m, k}$ replaced by $\mathcal{G}_{s, m, 0}$. Let $G^{o}(\alpha, x, y)$ be the $\alpha$-Green function corresponding to the ODGDO $\mathcal{G}_{s, m, 0}$. For $h \in \mathcal{M}_{s, m}$, we consider $p_{h}^{o}(t, x, y)$ given by (1.2) and set

$$
G_{h}^{o}(\alpha, x, y)=G^{o}(\alpha, x, y) / h(x) h(y), \quad x, y \in I .
$$

The following result is obtained by Maeno (see Theorem 2.2 of [4]).

PROPOSITION 3.1. [4] $p_{h}^{o}(t, x, y)$ is the elementary solution of $(1.1)$ with $\mathcal{G}_{s, m, k}$ replaced by $\mathcal{G}_{s_{h}, m_{h}, k_{h}}$, and $G_{h}^{o}(\alpha, x, y)$ is the $\alpha$-Green function corresponding to the ODGDO $\mathcal{G}_{s_{h}, m_{h}, k_{h}}$.

\section{2. $h$ transform of $\mathcal{G}_{s, m, k}$ with $h \in \mathcal{H}_{s, m, k, \beta}$}

For $\beta \geq 0$, let $h_{\beta}(\cdot)$ be a positive continuous function on $I$ satisfying

$$
\begin{aligned}
h_{\beta}(x)= & h_{\beta}\left(c_{o}\right)+D_{s} h_{\beta}\left(c_{o}\right)\left\{s(x)-s\left(c_{o}\right)\right\} \\
& +\int_{\left(c_{o}, x\right]}\{s(x)-s(y)\} h_{\beta}(y)\{\beta d m(y)+d k(y)\}, \quad x \in I .
\end{aligned}
$$

There exists such a function $h_{\beta}(\cdot)$. Indeed, it is represented as a linear combination of $g_{i}(\cdot, \beta), i=1,2$, given in the preceding section. Let $\mathcal{H}_{s, m, k, \beta}$ be the set of all positive functions $h_{\beta}$ satisfying (3.2). It immediately follows from (3.2) that

$$
\mathcal{H}_{s, m, k, \beta}=\mathcal{H}_{s, m, k+\beta m, 0}, \quad \beta \geq 0 .
$$

For $h \in \mathcal{H}_{s, m, k, \beta}$ and $g_{i}(x, \alpha), i=1,2$, satisfying (2.5)-(2.9), we set

$$
g_{h, i}(x, \alpha)=g_{i}(x, \alpha) / h(x), \quad i=1,2, \alpha \geq 0 .
$$

Let $G_{h}^{*}(\gamma, x, y)$ be the $\gamma$-Green function corresponding to $\mathcal{G}_{s_{h}, m_{h}, 0}$, where $s_{h}$ and $m_{h}$ are given by (1.3) and (1.4), respectively. Now we obtain the following proposition. Under the assumption $I_{*}(m)=I$, the following result is obtained as Proposition 2.2 and Lemma 3.3 of [8]. It is not difficult to see that their proofs are available without the assumption $I_{*}(m)=I$. Therefore we omit the proof of the following proposition.

Proposition 3.2. Let $\alpha \geq \beta \geq 0$ and $h \in \mathcal{H}_{s, m, k, \beta}$.

(i) Let $i=1$, 2. If $\alpha=\beta$ and $\left|s_{h}\left(l_{i}\right)\right|=\infty$, then $g_{h, i}(x, \alpha)$ is a positive constant function on I. If $\alpha>\beta$ or $\left|s_{h}\left(l_{i}\right)\right|<\infty$, then $g_{h, i}(x, \alpha)$ satisfies the following properties: 
(i-1) $g_{h, i}(x, \alpha)$ is positive and continuous on $I$;

(i-2) $g_{h, 1}(x, \alpha)$ is nondecreasing on $I$ and $g_{h, 2}(x, \alpha)$ is nonincreasing on I;

(i-3) if $\left|s_{h}\left(l_{i}\right)\right|<\infty$ or $\left|m_{h}\left(l_{i}\right)\right|=\infty$, then $g_{h, i}\left(l_{i}, \alpha\right)=0$;

(i-4) if $\left|s_{h}\left(l_{i}\right)\right|=\infty$, then $D_{s_{h}} g_{h, i}\left(l_{i}, \alpha\right)=0$;

(i-5) $g_{h, i}(x, \alpha)$ satisfies

$$
\begin{aligned}
g_{h, i}(x, \alpha)= & g_{h, i}\left(c_{o}, \alpha\right)+D_{s_{h}} g_{h, i}\left(c_{o}, \alpha\right)\left\{s_{h}(x)-s_{h}\left(c_{o}\right)\right\} \\
& +(\alpha-\beta) \int_{\left(c_{o}, x\right]}\left\{s_{h}(x)-s_{h}(y)\right\} g_{h, i}(y, \alpha) d m_{h}(y),
\end{aligned}
$$

for $x \in I$.

(ii) The following equation (3.5) holds true:

$$
\begin{aligned}
G_{h}^{*}(\alpha-\beta, x, y) & =G_{h}^{*}(\alpha-\beta, y, x) \\
& =W(\alpha)^{-1} g_{h, 1}(x, \alpha) g_{h, 2}(y, \alpha) \\
& =G(\alpha, x, y) / h(x) h(y),
\end{aligned}
$$

for $l_{1}<x \leq y<l_{2}$.

Let $p(t, x, y)$ be the elementary solution of equation (1.1) and consider $p_{h}^{*}(t, x, y)$ defined by (1.6). By virtue of (2.11) and (3.5), we get the following.

Proposition 3.3. $p_{h}^{*}(t, x, y)$ is the elementary solution of equation (1.1) with $\mathcal{G}_{s, m, k}$ replaced by $\mathcal{G}_{s_{h}, m_{h}, 0}$, and $G_{h}^{*}(\alpha, x, y)$ is the $\alpha$-Green function corresponding to $\mathcal{G}_{s_{h}, m_{h}, 0}$.

\subsection{Proof of Proposition 1.1}

(i) We set $\Lambda=\left\{h \in \mathcal{M}_{s, m} ; D_{s} h(x)\right.$ is a constant function on $\left.I\right\}$. It follows from (3.2) that

$$
D_{s} h(y)-D_{s} h(x)=\int_{(x, y]} h(z)\{\beta d m(z)+d k(z)\},
$$

for $h \in \mathcal{H}_{s, m, k, \beta}$. Therefore, $h$ belongs to $\mathcal{H}_{s, m, 0,0}$ if and only if $D_{s} h$ is a constant function, from which the set $\left\{x \in I ; D_{s} h\left(x_{1}\right)>D_{s} h\left(x_{2}\right)\right.$ for $\left.l_{1}<\forall x_{1}<x<\forall x_{2}<l_{2}\right\}$ is empty and hence it is included in $I_{*}(m)$. Thus $\mathcal{H}_{s, m, 0,0}$ is included in the set $\Lambda$. Conversely, $h \in \Lambda$ if and only if (3.6) holds true for the null killing measure and $\beta=0$, which implies (3.2) with $h=h_{\beta}, k=0$ and $\beta=0$. Therefore the set $\Lambda$ is included in $\mathcal{H}_{s, m, 0,0}$.

(ii) Assume that $k$ is not a null measure or $\beta>0$. Let $h \in \mathcal{H}_{s, m, k, \beta}$. By means of (3.6) we see that $D_{s} h(x)<D_{s} h(y)$ whenever $x<y$ and $(x, y] \cap I_{*}(m) \neq \emptyset$. Therefore $D_{s} h$ is increasing on $I_{*}(m)$ and it is nondecreasing on $I$. Thus $h$ does not belong to $\mathcal{M}_{s, m}$, which implies the second statement.

\subsection{Proofs of Theorem 1.2 and Corollary 1.3}

Proof of Theorem 1.2. Let $u$ be a measurable function on $I$. We show the first statement (i). Let $G^{o}(\alpha, x, y)$ be the $\alpha$-Green function corresponding to $\mathcal{G}_{s, m, 0}$. Let $h \in \mathcal{M}_{s, m}$ and consider $G_{h}^{o}(\alpha, x, y)$ given by (3.1). By means of (2.14) and Proposition 3.1, $u$ belongs to $D\left(\mathcal{G}_{s_{h}, m_{h}, k_{h}}\right)$ 
if and only if there is a function $f \in L^{2}\left(I, m_{h}\right)$ such that

$$
u(x)=\int_{I} G_{h}^{o}(\alpha, x, y) f(y) d m_{h}(y),
$$

or equivalently,

$$
u(x)=\frac{1}{h(x)} \int_{I} G^{o}(\alpha, x, y) f(y) h(y) d m(y) .
$$

We note that $f h$ belongs to $L^{2}(I, m)$. Therefore (3.8) holds true if and only if $h u$ belongs to $D\left(\mathcal{G}_{s, m, 0}\right)$. Proposition 3.1 combined with (3.7) and (3.8) implies

$$
u(x)=G_{h, \alpha}^{o} f(x)=\frac{1}{h(x)} G_{\alpha}^{o}(f h)(x),
$$

where $G_{h, \alpha}^{o}$ and $G_{\alpha}^{o}$ are the $\alpha$-Green operators corresponding to $\mathcal{G}_{s_{h}, m_{h}, k_{h}}$ and $\mathcal{G}_{s, m, 0}$, respectively. By means of (2.16),

$$
\left(\alpha-\mathcal{G}_{s_{h}, m_{h}, k_{h}}\right) G_{h, \alpha}^{o} f=f, \quad\left(\alpha-\mathcal{G}_{s, m, 0}\right) G_{\alpha}^{o}(f h)=f h,
$$

and hence we obtain

$$
\left(\alpha-\mathcal{G}_{s_{h}, m_{h}, k_{h}}\right) u=\frac{1}{h}\left(\alpha-\mathcal{G}_{s, m, 0}\right)(h u)
$$

that is,

$$
\mathcal{G}_{s_{h}, m_{h}, k_{h}} u=\frac{1}{h} \mathcal{G}_{s, m, 0}(h u)
$$

Thus we get statement (i).

Statement (ii) is obtained in the same way as above and so we omit the proof.

Proof of Corollary 1.3. Statement (i) immediately follows from Proposition 1.1 and Theorem 1.2.

(ii) Let $h \in \mathcal{M}_{s, m}$. By means of Theorem $1.2, H_{h}^{o} \mathcal{G}_{s, m, 0}=\mathcal{G}_{s, m, 0}$ if and only if $k_{h}$ is the null measure, and $s_{h}(x)=A s(x)+A_{1}, m_{h}(x)=A^{-1} m(x)+A_{2}$ for some positive constant $A$ and $A_{i} \in \mathbb{R}, i=1,2$, or equivalently $h$ is a positive constant function.

(iii) Let $h \in \mathcal{H}_{s, m, k, \beta}$. By means of Theorem 1.2, $H_{h}^{*} \mathcal{G}_{s, m, k+\beta m}=\mathcal{G}_{s, m, k+\beta m}$ if and only if $k+\beta m$ is the null measure, and $s_{h}(x)=B s(x)+B_{1}, m_{h}(x)=B^{-1} m(x)+B_{2}$ for some positive constant $B$ and $B_{i} \in \mathbb{R}, i=1,2$. This is equivalent to $k$ being the null measure, $\beta=0$, and $h$ being a positive constant function.

\subsection{Proof of Theorem 1.4}

(i) Let $h \in \mathcal{M}_{s, m}$. Then $D_{s_{h}} h^{-1}=-D_{s} h$ and hence

$$
\begin{aligned}
D_{s_{h}} h^{-1}(y)-D_{s_{h}} h^{-1}(x) & =-D_{s} h(y)+D_{s} h(x) \\
& =-\int_{(x, y]} d D_{s} h(z)=\int_{(x, y]} h^{-1}(z) d k_{h}(z) .
\end{aligned}
$$

This shows that $h^{-1}$ belongs to $\mathcal{H}_{s_{h}, m_{h}, k_{h}, 0}$. By means of Theorem 1.2(ii), $H_{h^{-1}}^{*} \mathcal{G}_{s_{h}, m_{h}, k_{h}}=$ $\mathcal{G}_{s, m, 0}$, from which $H_{h^{-1}}^{*} H_{h}^{o} \mathcal{G}_{s, m, 0}=\mathcal{G}_{s, m, 0}$. 
(ii) Let $h \in \mathcal{H}_{s, m, k, \beta}$. Then $D_{s_{h}} h^{-1}=-D_{s} h$, and by means of (3.6),

$$
\begin{aligned}
D_{s_{h}} h^{-1}(y)-D_{s_{h}} h^{-1}(x) & =-D_{s} h(y)+D_{s} h(x) \\
& =-\int_{(x, y]} h(z)\{\beta d m(z)+d k(z)\} .
\end{aligned}
$$

Therefore $D_{s_{h}} h^{-1}$ is nonincreasing on $I$ and the set $\Lambda=\left\{x \in I: D_{s_{h}} h^{-1}\left(x_{1}\right)>\right.$ $D_{s_{h}} h^{-1}\left(x_{2}\right)$ for $\left.l_{1}<\forall x_{1}<x<\forall x_{2}<l_{2}\right\}$ is included in $I_{*}(m) \cup I_{*}(k)$. Since $I_{*}(m) \cup$ $I_{*}(k)=I_{*}(m)=I_{*}\left(m_{h}\right)$, we get $h^{-1} \in \mathcal{M}_{s_{h}, m_{h}}$. Calculating the right-hand side of (1.5) with $s$ and $h$ replaced by $s_{h}$ and $h^{-1}$, respectively, we see by virtue of (3.9) that

$$
-\int_{\left(c_{o}, x\right]} h^{-1}(y) d D_{s_{h}} h^{-1}(y)=\int_{\left(c_{o}, x\right]}\{\beta d m(y)+d k(y)\} .
$$

Combining this with Theorem 1.2(i), we get $H_{h^{-1}}^{o} \mathcal{G}_{s_{h}, m_{h}, 0}=\mathcal{G}_{s, m, k+\beta m}$, from which $H_{h^{-1}}^{o} H_{h}^{*} \mathcal{G}_{s, m, k+\beta m}=\mathcal{G}_{s, m, k+\beta m}$.

\section{Global properties of ODGDOs and their $h$ transform}

In this section we show Theorem 1.5. First we prepare two lemmas on properties of $h \in \mathcal{M}_{s, m}$ and $h \in \mathcal{H}_{s, m, k, \beta}$.

Lemma 4.1. Assume that $s\left(l_{1}\right)=-\infty$ and $s\left(l_{2}\right)=\infty$. If $h \in \mathcal{M}_{s, m}$ and $k_{h}$ is the null measure, then $h$ is a positive constant function. If $h \in \mathcal{H}_{s, m, 0, \beta}, s_{h}\left(l_{1}\right)=-\infty$ and $s_{h}\left(l_{2}\right)=\infty$, then $h$ is a positive constant function and $\beta=0$.

Proof. Assume that $s\left(l_{1}\right)=-\infty$ and $s\left(l_{2}\right)=\infty$.

(i) Suppose that $h \in \mathcal{M}_{s, m}$ and $k_{h}$ is the null measure. By means of (1.5), $D_{s} h$ is a constant function. Therefore $h(x)$ is represented as $h(x)=C_{1} s(x)+C_{2}$ for some $C_{i} \in$ $\mathbb{R}, i=1,2$. Since $s\left(l_{1}\right)=-\infty, s\left(l_{2}\right)=\infty$ and $h(x)$ is a positive function, we get $C_{1}=0$ and $C_{2}>0$.

(ii) Suppose that $h \in \mathcal{H}_{s, m, 0, \beta}, s_{h}\left(l_{1}\right)=-\infty$ and $s_{h}\left(l_{2}\right)=\infty$. By using Lemma 3.2(i) of [8], we see that $h\left(l_{i}\right)<\infty, i=1,2$. Note that $h(x)$ is represented as $h(x)=C_{3} g_{1}(x, \beta)+$ $C_{4} g_{2}(x, \beta)$, where $g_{i}(x, \beta), i=1,2$, are functions satisfying (2.5)-(2.9) with $\alpha=\beta$ and $k=0$. If $\beta>0$, by means of Proposition 2.1(i), $g_{i}\left(l_{i}, \beta\right)<\infty$ for $i=1,2$, and $g_{i}\left(l_{j}, \beta\right)=\infty$ for $i \neq j$. Combining these with $h\left(l_{i}\right)<\infty$ for $i=1,2$, we see $C_{3}=C_{4}=0$, and hence $h(x)=0$. This contradicts $h(x)>0$ on $I$. Thus $\beta=0$. Noting $s\left(l_{1}\right)=-\infty, s\left(l_{2}\right)=\infty$ and Proposition 2.1(ii), we obtain that $h$ is a positive constant function.

LEMMA 4.2.

(i) Let $h \in \mathcal{M}_{s, m}$. If $s\left(l_{1}\right)>-\infty$ or $s\left(l_{2}\right)<\infty$, then $s_{h}\left(l_{1}\right)>-\infty$, or $s_{h}\left(l_{2}\right)<\infty$, or $k_{h}$ is not a null measure.

(ii) Let $h \in \mathcal{H}_{s, m, k, \beta}$. If $s\left(l_{1}\right)>-\infty$, or $s\left(l_{2}\right)<\infty$, or $k$ is not a null measure, then $s_{h}\left(l_{1}\right)>-\infty$ or $s_{h}\left(l_{2}\right)<\infty$.

Proof. (i) Let $h \in \mathcal{M}_{s, m}$, and $s\left(l_{1}\right)>-\infty$ or $s\left(l_{2}\right)<\infty$. It is enough to show that $s_{h}\left(l_{1}\right)>$ $-\infty$ or $s_{h}\left(l_{2}\right)<\infty$ in the case that $k_{h}$ is the null measure. Since $k_{h}$ is the null measure, $D_{s} h$ is a constant function by virtue of (1.5). 
Suppose $s\left(l_{1}\right)>-\infty$. By means of Lemma 2.1(ii) of [4], we have $h\left(l_{1}\right) \in[0, \infty)$. If $h\left(l_{1}\right) \in(0, \infty)$, then $s_{h}\left(l_{1}\right)>-\infty$. Let $h\left(l_{1}\right)=0$. Noting that $D_{s} h$ is a constant function, we see that $h(x)=C_{1}\left\{s(x)-s\left(l_{1}\right)\right\}$ for some $C_{1}>0$. Equation (1.3) coupled with this implies $s_{h}\left(l_{2}\right)<\infty$.

In the same way as above, we get $s_{h}\left(l_{1}\right)>-\infty$ or $s_{h}\left(l_{2}\right)<\infty$ when $s\left(l_{2}\right)<\infty$.

(ii) Let $h \in \mathcal{H}_{s, m, k, \beta}$ and assume that $s\left(l_{1}\right)>-\infty$, or $s\left(l_{2}\right)<\infty$, or $k$ is not a null measure. Note $h(x)$ is represented as $h(x)=C_{2} g_{1}(x, \beta)+C_{3} g_{2}(x, \beta)$, where $g_{i}(x, \beta), i=$ 1,2 , are functions satisfying (2.5)-(2.9) with $\alpha=\beta$. We divide the proof into four cases.

Case 1: $h\left(l_{1}\right)=\infty$. Then $s_{h}\left(l_{1}\right)>-\infty$ by virtue of Lemma 3.2(i) of [8].

Case 2: $h\left(l_{1}\right) \in(0, \infty)$ and $s\left(l_{1}\right)>-\infty$. Then $s_{h}\left(l_{1}\right)>-\infty$ by means of (1.3).

Case 3: $h\left(l_{1}\right) \in(0, \infty)$ with $s\left(l_{1}\right)=-\infty$. Since $g_{2}\left(l_{1}, \beta\right)=\infty$ for $\beta \geq 0$ by virtue of Proposition 2.1, we get $C_{3}=0$ and $h(x)=C_{2} g_{1}(x, \beta)$.

(3-1) Let $s\left(l_{2}\right)<\infty$. Then

$$
s_{h}\left(l_{2}\right)=\int_{\left(c_{o}, l_{2}\right)} h(y)^{-2} d s(y) \leq C_{2}^{-2} g_{1}\left(c_{o}, \beta\right)^{-2}\left\{s\left(l_{2}\right)-s\left(c_{o}\right)\right\}<\infty .
$$

(3-2) Let $s\left(l_{2}\right)=\infty$. Since $k$ is not a null measure, we have, by virtue of Proposition 2.1(i), $h\left(l_{2}\right)=C_{2} g_{1}\left(l_{2}, \beta\right)=\infty$ for $\beta \geq 0$. Therefore $s_{h}\left(l_{2}\right)<\infty$ by virtue of Lemma 3.2(i) of [8].

Case 4: $h\left(l_{1}\right)=0$. Then $h(x)$ is represented as $h(x)=C_{2} g_{1}(x, \beta)$, because of $g_{2}\left(l_{1}, \beta\right)>0$. Thus we have $s_{h}\left(l_{2}\right)<\infty$ in the same way as in (3-1) and (3-2).

Proof of Theorem 1.5. (i) Let $\mathcal{G}_{s, m, 0} \in \Phi^{R}$. Hence $s\left(l_{1}\right)=-\infty$ and $s\left(l_{2}\right)=\infty$.

Suppose that $H_{h}^{o} \mathcal{G}_{s, m, 0}=\mathcal{G}_{s_{h}, m_{h}, k_{h}} \in \Phi^{R}$ for some $h \in \mathcal{M}_{s, m}$. Then $k_{h}$ is the null measure. By virtue of Lemma 4.1, $h$ is a positive constant function.

Suppose that $H_{h}^{*} \mathcal{G}_{s, m, \beta m}=\mathcal{G}_{s_{h}, m_{h}, 0} \in \Phi^{R}$ for some $h \in \mathcal{H}_{s, m, 0, \beta}$. Then $s_{h}\left(l_{1}\right)=-\infty$ and $s_{h}\left(l_{2}\right)=\infty$. By virtue of Lemma $4.1, h$ is a positive constant function and $\beta=0$.

(ii) The former statement follows from Corollary 1.3. The later follows from Lemma 4.1.

(iii) (1) Let $\mathcal{G}_{s, m, 0} \in \Phi^{T}$ and $h \in \mathcal{M}_{s, m}$. Since $s\left(l_{1}\right)>-\infty$ or $s\left(l_{2}\right)<\infty$, by means of Lemma $4.2(\mathrm{i}), s_{h}\left(l_{1}\right)>-\infty$ or $s_{h}\left(l_{2}\right)<\infty$ or $k_{h}$ is not a null measure. Thus $H_{h}^{o} \mathcal{G}_{s, m, 0}=$ $\mathcal{G}_{s_{h}, m_{h}, k_{h}} \in \Phi^{T}$.

(2) Let $\mathcal{G}_{s, m, k} \in \Phi^{T}$ and $h \in \mathcal{H}_{s, m, k, \beta}$. Noting that $s\left(l_{1}\right)>-\infty$, or $s\left(l_{2}\right)<\infty$, or $k$ is not a null measure, and using Lemma 4.2(ii), we see $s_{h}\left(l_{1}\right)>-\infty$ or $s_{h}\left(l_{2}\right)<\infty$. This implies $H_{h}^{*} \mathcal{G}_{s, m, k+\beta m}=\mathcal{G}_{s_{h}, m_{h}, 0} \in \Phi^{T}$.

\section{Spectral representation of the elementary solution}

Let $\mathcal{G}_{s, m, k}$ be an ODGDO and let $p(t, x, y)$ be the elementary solution of equation (1.1). We assume that $I_{*}(m)=I$ and $l_{1}$ is not $(s, m, k)$-natural. Further, we assume that $p(t, x, y)$ is represented as (1.7). Namely,

$$
p(t, x, y)=\int_{[0, \infty)} e^{-\lambda t} \psi(x, \lambda) \psi(y, \lambda) d \sigma(\lambda), \quad t>0, x, y \in I,
$$


where $d \sigma(\lambda)$ is a Borel measure on $[0, \infty) ; \sigma(\{0\})=0$ if $l_{1}$ is $(s, m, k)$-regular or -exit. Further, $\psi(x, \lambda), x \in I, \lambda \geq 0$, is the solution of the integral equation

$$
\psi(x, \lambda)=\left\{\begin{aligned}
& s(x)-s\left(l_{1}\right)+\int_{\left(l_{1}, x\right]}\{s(x)-s(y)\} \psi(y, \lambda)\{-\lambda d m(y)+d k(y)\}, \\
& \text { if } l_{1} \text { is }(s, m, k) \text {-regular or -exit, } \\
& 1+\int_{\left(l_{1}, x\right]}\{s(x)-s(y)\} \psi(y, \lambda)\{-\lambda d m(y)+d k(y)\}, \\
& \quad \text { if } l_{1} \text { is }(s, m, k) \text {-entrance. }
\end{aligned}\right.
$$

In the following we fix an $h \in \mathcal{H}_{s, m, k, \beta}$ arbitrarily. Then $\mathcal{G}_{s_{h}, m_{h}, 0}$ is an ODGDO and $p_{h}^{*}(t, x, y)$ given by (1.6) is the elementary solution of equation (1.1) with $\mathcal{G}_{s_{h}, m_{h}, 0}$ in place of $\mathcal{G}_{s, m, k}$. We set $\psi_{h}(x, \lambda)=\psi(x, \lambda) / h(x)$. By virtue of (1.6) and (1.7), we obtain

$$
\begin{aligned}
p_{h}^{*}(t, x, y) & =e^{-\beta t} \frac{p(t, x, y)}{h(x) h(y)} \\
& =\int_{[\beta, \infty)} e^{-\lambda t} \psi_{h}(x, \lambda-\beta) \psi_{h}(y, \lambda-\beta) d \sigma_{\lambda}(\lambda-\beta) .
\end{aligned}
$$

In the same way as in the proof of Lemma 3.3 of [8], we see that $\psi_{h}(x, \lambda)$ satisfies

$$
D_{s_{h}} \psi_{h}(y, \lambda)-D_{s_{h}} \psi_{h}(x, \lambda)=-(\lambda+\beta) \int_{(x, y]} \psi_{h}(z, \lambda) d m_{h}(z)
$$

for $l_{1}<x \leq y<l_{2}$. We note the following lemma.

LEMMA 5.1.

(i) Let $l_{1}$ be $(s, m, k)$-regular or -exit. Then $h\left(l_{1}\right) \in[0, \infty)$ and

$$
\begin{aligned}
& \psi_{h}\left(l_{1}, \lambda\right)=D_{s} h\left(l_{1}\right)^{-1} \in(0, \infty), \quad D_{s_{h}} \psi_{h}\left(l_{1}, \lambda\right)=0, \quad \text { if } h\left(l_{1}\right)=0 ; \\
& \psi_{h}\left(l_{1}, \lambda\right)=0, \quad D_{s_{h}} \psi_{h}\left(l_{1}, \lambda\right)=h\left(l_{1}\right) \in(0, \infty), \quad \text { if } h\left(l_{1}\right) \in(0, \infty) .
\end{aligned}
$$

(ii) Let $l_{1}$ be $(s, m, k)$-entrance. Then $h\left(l_{1}\right) \in(0, \infty]$ and

$$
\begin{gathered}
\psi_{h}\left(l_{1}, \lambda\right)=h\left(l_{1}\right)^{-1} \in(0, \infty), \quad D_{s_{h}} \psi_{h}\left(l_{1}, \lambda\right)=0, \quad \text { if } h\left(l_{1}\right) \in(0, \infty) ; \\
\psi_{h}\left(l_{1}, \lambda\right)=0, \quad D_{s_{h}} \psi_{h}\left(l_{1}, \lambda\right)=-D_{s} h\left(l_{1}\right) \in(0, \infty), \quad \text { if } h\left(l_{1}\right)=\infty .
\end{gathered}
$$

Proof. Note that $h$ is represented as $h(x)=C_{1} g_{1}(x, \beta)+C_{2} g_{2}(x, \beta)$, where $g_{i}(x, \beta)$, $i=1,2$, are functions satisfying (2.5)-(2.9) with $\alpha=\beta$.

(i) Let $l_{1}$ be $(s, m, k)$-regular or -exit. By means of Theorem 1.1 of [7], $0 \leq h\left(l_{1}\right)<\infty$.

First we consider the case $h\left(l_{1}\right)=0$, and hence $C_{1}>0$ and $C_{2}=0$, by virtue of Proposition 2.1. Since $D_{s} h\left(l_{1}\right)=C_{1} D_{s} g_{1}\left(l_{1}, \beta\right) \in(0, \infty)$, we get

$$
\begin{aligned}
\psi_{h}\left(l_{1}, \lambda\right) & =\lim _{x \rightarrow l_{1}} \frac{\psi(x, \lambda)}{h(x)}=\lim _{x \rightarrow l_{1}} \frac{D_{s} \psi(x, \lambda)}{D_{s} h(x)}=\frac{1}{D_{s} h\left(l_{1}\right)} \in(0, \infty), \\
D_{s_{h}} \psi_{h}\left(l_{1}, \lambda\right) & =\lim _{x \rightarrow l_{1}}\left\{h(x) D_{s} \psi(x, \lambda)-\psi(x, \lambda) D_{s} h(x)\right\}=0 .
\end{aligned}
$$

Thus we obtain (5.5). 
Next we consider the case $0<h\left(l_{1}\right)<\infty$. Then $C_{2}>0$ and $\psi_{h}\left(l_{1}, \lambda\right)=\psi\left(l_{1}, \lambda\right) / h\left(l_{1}\right)$ $=0$. If $l_{1}$ is $(s, m, k)$-regular, then by means of Proposition 2.1, $D_{s} h\left(l_{1}\right)=C_{1} D_{s} g_{1}\left(l_{1}, \beta\right)+$ $C_{2} D_{s} g_{2}\left(l_{1}, \beta\right) \in \mathbb{R}$ and hence

$$
D_{s_{h}} \psi_{h}\left(l_{1}, \lambda\right)=\lim _{x \rightarrow l_{1}}\left\{h(x) D_{s} \psi(x, \lambda)-\psi(x, \lambda) D_{s} h(x)\right\}=h\left(l_{1}\right) \in(0, \infty) .
$$

If $l_{1}$ is $(s, m, k)$-exit, then by means of Proposition 2.1

$$
\begin{aligned}
\lim _{x \rightarrow l_{1}} \frac{\psi(x, \lambda)}{g_{1}(x, \beta)} & =\lim _{x \rightarrow l_{1}} \frac{D_{s} \psi(x, \lambda)}{D_{s} g_{1}(x, \beta)}=\frac{1}{D_{s} g_{1}\left(l_{1}, \beta\right)} \in(0, \infty), \\
\lim _{x \rightarrow l_{1}} \psi(x, \lambda) D_{s} h(x) & =\frac{1}{D_{s} g_{1}\left(l_{1}, \beta\right)} \lim _{x \rightarrow l_{1}} g_{1}(x, \beta)\left\{C_{1} D_{s} g_{1}(x, \beta)+C_{2} D_{s} g_{2}(x, \beta)\right\}=0 .
\end{aligned}
$$

Therefore we arrive at

$$
\begin{aligned}
D_{s_{h}} \psi_{h}\left(l_{1}, \lambda\right) & =\lim _{x \rightarrow l_{1}}\left\{h(x) D_{s} \psi(x, \lambda)-\psi(x, \lambda) D_{s} h(x)\right\} \\
& =h\left(l_{1}\right) \in(0, \infty) .
\end{aligned}
$$

Thus we have (5.6).

(ii) Let $l_{1}$ be $(s, m, k)$-entrance. By means of Theorem 1.1 of [7], $0<h\left(l_{1}\right) \leq \infty$.

First we consider the case $0<h\left(l_{1}\right)<\infty$, and hence $C_{1}>0$ and $C_{2}=0$ by virtue of Proposition 2.1. Then

$$
\begin{aligned}
\psi_{h}\left(l_{1}, \lambda\right) & =\frac{1}{h\left(l_{1}\right)}=\frac{1}{C_{1} g_{1}\left(l_{1}, \beta\right)} \in(0, \infty), \\
D_{s_{h}} \psi_{h}\left(l_{1}, \lambda\right) & =h\left(l_{1}\right) D_{s} \psi\left(l_{1}, \lambda\right)-\psi\left(l_{1}, \lambda\right) D_{s} h\left(l_{1}\right)=-C_{1} D_{s} g_{1}\left(l_{1}, \beta\right)=0 .
\end{aligned}
$$

These show (5.7).

Next we consider the case $h\left(l_{1}\right)=\infty$. Then $C_{2}>0$ and $\psi_{h}\left(l_{1}, \lambda\right)=\psi\left(l_{1}, \lambda\right) / h\left(l_{1}\right)=0$. We show that

$$
D_{s_{h}} \psi_{h}\left(l_{1}, \lambda\right)=-D_{s} h\left(l_{1}\right)=-C_{2} D_{s} g_{2}\left(l_{1}, \beta\right) \in(0, \infty) .
$$

Here is the proof of (5.9). Since $l_{1}$ is $(s, m, k)$-entrance, for any positive $\varepsilon$ there exists an $r \in I$ such that

$$
\begin{aligned}
\{s(r)-s(x)\} \int_{\left(l_{1}, x\right]}(\lambda d m(z)+d k(z)) & \leq \int_{\left(l_{1}, x\right]}\{s(r)-s(z)\}(\lambda d m(z)+d k(z)) \\
& \leq \int_{\left(l_{1}, r\right]}\{s(r)-s(z)\}(\lambda d m(z)+d k(z))<\varepsilon,
\end{aligned}
$$

for $l_{1}<x<r$. It is easy to derive the following estimate from (5.2):

$$
\left|D_{s} \psi(x, \lambda)\right| \leq \int_{\left(l_{1}, x\right]}(\lambda d m(z)+d k(z)) \exp \left\{\int_{\left(l_{1}, x\right]}(\lambda d m(z)+d k(z)) \int_{(z, x]} d s(y)\right\} .
$$

Combining this with (5.10), we find

$$
\limsup _{x \rightarrow l_{1}}\{s(r)-s(x)\}\left|D_{s} \psi(x, \lambda)\right|<\varepsilon .
$$


Therefore

$$
\limsup _{x \rightarrow l_{1}} h(x)\left|D_{s} \psi(x, \lambda)\right| \leq \varepsilon \lim _{x \rightarrow l_{1}} \frac{h(x)}{s(r)-s(x)}=-\varepsilon D_{s} h\left(l_{1}\right)=-\varepsilon C_{2} D_{s} g_{2}\left(l_{1}, \beta\right) .
$$

Since $D_{s} g_{2}\left(l_{1}, \beta\right) \in(-\infty, 0)$ by virtue of Proposition 2.1, letting $\varepsilon \downarrow 0$ leads us to $\lim _{x \rightarrow l_{1}} h(x) D_{s} \psi(x, \lambda)=0$, and

$$
\begin{aligned}
D_{s_{h}} \psi_{h}\left(l_{1}, \lambda\right) & =\lim _{x \rightarrow l_{1}}\left\{h(x) D_{s} \psi(x, \lambda)-\psi(x, \lambda) D_{s} h(x)\right\} \\
& =-D_{s} h\left(l_{1}\right)=-C_{2} D_{s} g_{2}\left(l_{1}, \beta\right) \in(0, \infty),
\end{aligned}
$$

which shows (5.9). Thus we obtain (5.8).

Now we define $\psi_{h}^{*}(x, \lambda), x \in I, \lambda \geq \beta$, and $d \sigma_{h}^{*}(\lambda), \lambda \geq \beta$, as follows.

Case 1: $l_{1}$ is (s, $\left.m, k\right)$-regular or -exit. If $h\left(l_{1}\right)=0$, then

$$
\begin{aligned}
\psi_{h}^{*}(x, \lambda) & =D_{s} h\left(l_{1}\right) h(x)^{-1} \psi(x, \lambda-\beta), \\
d \sigma_{h}^{*}(\lambda) & =\left\{D_{s} h\left(l_{1}\right)\right\}^{-2} d_{\lambda} \sigma(\lambda-\beta) .
\end{aligned}
$$

If $h\left(l_{1}\right) \in(0, \infty)$, then

$$
\begin{aligned}
\psi_{h}^{*}(x, \lambda) & =\left\{h\left(l_{1}\right) h(x)\right\}^{-1} \psi(x, \lambda-\beta), \\
d \sigma_{h}^{*}(\lambda) & =h\left(l_{1}\right)^{2} d_{\lambda} \sigma(\lambda-\beta) .
\end{aligned}
$$

Case 2: $l_{1}$ is $(s, m, k)$-entrance. If $h\left(l_{1}\right) \in(0, \infty)$, then

$$
\begin{aligned}
\psi_{h}^{*}(x, \lambda) & =h\left(l_{1}\right) h(x)^{-1} \psi(x, \lambda-\beta), \\
d \sigma_{h}^{*}(\lambda) & =h\left(l_{1}\right)^{-2} d_{\lambda} \sigma(\lambda-\beta) .
\end{aligned}
$$

If $h\left(l_{1}\right)=\infty$, then

$$
\begin{aligned}
\psi_{h}^{*}(x, \lambda) & =\left\{-D_{s} h\left(l_{1}\right) h(x)\right\}^{-1} \psi(x, \lambda-\beta), \\
d \sigma_{h}^{*}(\lambda) & =\left\{D_{s} h\left(l_{1}\right)\right\}^{2} d_{\lambda} \sigma(\lambda-\beta) .
\end{aligned}
$$

We note that $\sigma_{h}^{*}(\{\beta\})=0$ in Case 1 , but $\sigma_{h}^{*}(\{\beta\}) \geq 0$ in Case 2 .

By means of Theorem 1.1 of [7], $l_{1}$ is not $\left(s_{h}, m_{h}, 0\right)$-natural. More precisely, in the case $l_{1}$ is $(s, m, k)$-regular (respectively -exit),

$$
\begin{array}{ll}
l_{1} \text { is }\left(s_{h}, m_{h}, 0\right) \text {-regular (respectively -exit) } & \text { if } h\left(l_{1}\right) \in(0, \infty), \\
l_{1} \text { is }\left(s_{h}, m_{h}, 0\right) \text {-entrance } & \text { if } h\left(l_{1}\right)=0 ;
\end{array}
$$

in the case $l_{1}$ is $(s, m, k)$-entrance,

$$
\begin{array}{ll}
l_{1} \text { is }\left(s_{h}, m_{h}, 0\right) \text {-entrance } & \text { if } h\left(l_{1}\right) \in(0, \infty), \\
l_{1} \text { is }\left(s_{h}, m_{h}, 0\right) \text {-regular } & \text { if } h\left(l_{1}\right)=\infty \text { and }\left|m_{h}\left(l_{1}\right)\right|<\infty, \\
l_{1} \text { is }\left(s_{h}, m_{h}, 0\right) \text {-exit } & \text { if } h\left(l_{1}\right)=\infty \text { and }\left|m_{h}\left(l_{1}\right)\right|=\infty .
\end{array}
$$

Therefore (5.3), (5.4) and Lemma 5.1 lead us to the following result.

PROPOSITION 5.2. $\psi_{h}^{*}(x, \lambda)$ satisfies (5.1) or (5.2) with $s, m$ and $k$ replaced by $s_{h}, m_{h}$ and 0 , respectively. $d \sigma_{h}^{*}(\lambda)$ is a Borel measure on $[\beta, \infty) . p_{h}^{*}(t, x, y)$ is represented as

$$
p_{h}^{*}(t, x, y)=\int_{[0, \infty)} e^{-\lambda t} \psi_{h}^{*}(x, \lambda) \psi_{h}^{*}(y, \lambda) d \sigma_{h}^{*}(\lambda), \quad t>0, x, y \in I .
$$




\section{Examples}

Example 6.1. First we consider the following ODGDO $\mathcal{G}_{s, m, k}$ on $I=(0, \infty)$ with constant coefficients:

$$
\mathcal{G}_{s, m, k}=a \frac{d^{2}}{d x^{2}}+b \frac{d}{d x}-c,
$$

where $a>0, b \in \mathbb{R}$ and $c \geq 0$. We may set

$$
d s(x)=e^{-(b / a) x} d x, \quad d m(x)=a^{-1} e^{(b / a) x} d x, \quad d k(x)=(c / a) e^{(b / a) x} d x .
$$

The end point 0 is $(s, m, k)$-regular and the end point $\infty$ is $(s, m, k)$-natural. For $\alpha \geq 0$, we set

$$
\lambda_{i}(\alpha)=\frac{1}{2 a}\left\{(-1)^{i} b+\sqrt{b^{2}+4 a(c+\alpha)}\right\}, \quad i=1,2 .
$$

The $\alpha$-Green function $G(\alpha, x, y)$ corresponding to $\mathcal{G}_{s, m, k}$ is given by

$$
G(\alpha, x, y)=G(\alpha, y, x)= \begin{cases}\left(\lambda_{1}(\alpha)+\lambda_{2}(\alpha)\right)^{-1} g_{1}(x, \alpha) g_{2}(y, \alpha), & \text { if } b^{2}+c+\alpha>0, \\ g_{1}(x, \alpha) g_{2}(y, \alpha), & \text { if } b^{2}+c+\alpha=0,\end{cases}
$$

for $0 \leq x \leq y<\infty$, where

$$
\begin{aligned}
& g_{1}(x, \alpha)= \begin{cases}e^{\lambda_{1}(\alpha) x}-e^{-\lambda_{2}(\alpha) x}, & \text { if } b^{2}+c+\alpha>0, \\
x, & \text { if } b^{2}+c+\alpha=0,\end{cases} \\
& g_{2}(x, \alpha)= \begin{cases}e^{-\lambda_{2}(\alpha) x}, & \text { if } b^{2}+c+\alpha>0, \\
1, & \text { if } b^{2}+c+\alpha=0 .\end{cases}
\end{aligned}
$$

The elementary solution $p(t, x, y)$ is given by

$$
\begin{aligned}
p(t, x, y) & =\frac{1}{2} \sqrt{\frac{a}{\pi t}} \exp \left\{-\frac{b}{2 a}(x+y)-A t\right\}\left\{e^{-(x-y)^{2} / 4 a t}-e^{-(x+y)^{2} / 4 a t}\right\} \\
& =\int_{A}^{\infty} e^{-\lambda t} \psi(x, \lambda) \psi(y, \lambda) d \sigma(\lambda),
\end{aligned}
$$

where $A=b^{2} / 4 a+c$ and

$$
\begin{aligned}
\psi(x, \lambda) & =e^{-(b / 2 a) x} \sqrt{\frac{a}{\lambda-A}} \sin \left(\sqrt{\frac{\lambda-A}{a}} x\right), \\
d \sigma(\lambda) & =\frac{1}{\pi} \sqrt{\frac{\lambda-A}{a}} d \lambda,
\end{aligned}
$$

for $x \in I$ and $\lambda>A$.

Let $h \in \mathcal{H}_{s, m, k, \beta}$. Then the $h$ transform of $\mathcal{G}_{s, m, k}$ is reduced to

$$
\mathcal{G}_{s_{h}, m_{h}, 0}=a \frac{d^{2}}{d x^{2}}+\left(b+2 a \frac{h^{\prime}(x)}{h(x)}\right) \frac{d}{d x} .
$$

(1) Assume $h(0) \in(0, \infty)$. Then $h$ is represented as $h(x)=C_{1} g_{1}(x, \beta)+C_{2} g_{2}(x, \beta)$ for some constants $C_{1} \geq 0$ and $C_{2}>0$. The end point 0 is $\left(s_{h}, m_{h}, 0\right)$-regular and we 
get (5.19), where by means of (5.13) and (5.14),

$$
\psi_{h}^{*}(x, \lambda)=\left\{C_{2} h(x)\right\}^{-1} \psi(x, \lambda-\beta), \quad d \sigma_{h}^{*}(\lambda)=C_{2}^{2} d_{\lambda} \sigma(\lambda-\beta) .
$$

(2) Assume $h(0)=0$. Then $h(x)=C_{1} g_{1}(x, \beta)$ with $C_{1}>0$. The end point 0 is $\left(s_{h}, m_{h}, 0\right)$-entrance and we get (5.19), where by means of (5.11) and (5.12),

$$
\psi_{h}^{*}(x, \lambda)=\widetilde{C} h(x)^{-1} \psi(x, \lambda-\beta), \quad d \sigma_{h}^{*}(\lambda)=\widetilde{C}^{-2} d_{\lambda} \sigma(\lambda-\beta) .
$$

Here

$$
\widetilde{C}=D_{s} h(0)= \begin{cases}C_{1}\left(\lambda_{1}(\beta)+\lambda_{2}(\beta)\right), & \text { if } b^{2}+c+\beta>0, \\ C_{1}, & \text { if } b^{2}+c+\beta=0 .\end{cases}
$$

Especially, if $b=c=0$ and $C_{1}=1$, then $\lambda_{i}(\beta)=\sqrt{\beta / a}, i=1,2$, and

$$
h(x)=\left\{\begin{array}{ll}
2 \sinh (\sqrt{\beta / a} x), & \text { if } \beta>0, \\
x, & \text { if } \beta=0,
\end{array} \quad \widetilde{C}= \begin{cases}2 \sqrt{\beta / a}, & \text { if } \beta>0, \\
1, & \text { if } \beta=0 .\end{cases}\right.
$$

Therefore (6.3) and (5.19) are reduced to the following. If $\beta>0$, then

$$
\begin{aligned}
\mathcal{G}_{s_{h}, m_{h}, 0} & =a \frac{d^{2}}{d x^{2}}+2 \sqrt{a \beta} \frac{\cosh (\sqrt{\beta / a} x)}{\sinh (\sqrt{\beta / a} x)} \frac{d}{d x}, \\
p_{h}^{*}(t, x, y) & =\frac{1}{8} \sqrt{\frac{a}{\pi t}} e^{-\beta t}\left\{e^{-(x-y)^{2} / 4 a t}-e^{-(x+y)^{2} / 4 a t}\right\} / \sinh (\sqrt{\beta / a} x) \sinh (\sqrt{\beta / a} y) \\
& =\int_{\beta}^{\infty} e^{-\lambda t} \psi_{h}^{*}(x, \lambda) \psi_{h}^{*}(y, \lambda) d \sigma_{h}^{*}(\lambda),
\end{aligned}
$$

where

$$
\psi_{h}^{*}(x, \lambda)=\sqrt{\frac{\beta}{\lambda-\beta}} \frac{\sin (\sqrt{(\lambda-\beta) / a} x)}{\sinh (\sqrt{\beta / a} x)}, \quad d \sigma_{h}^{*}(\lambda)=\frac{1}{4 \pi} \frac{\sqrt{(\lambda-\beta) / \beta}}{\sqrt{\beta / a}} d \lambda .
$$

If $\beta=0$, then

$$
\begin{aligned}
\mathcal{G}_{s_{h}, m_{h}, 0} & =a \frac{d^{2}}{d x^{2}}+\frac{2 a}{x} \frac{d}{d x}, \\
p_{h}^{*}(t, x, y) & =\frac{1}{2} \sqrt{\frac{a}{\pi t}} \frac{1}{x y}\left\{e^{-(x-y)^{2} / 4 a t}-e^{-(x+y)^{2} / 4 a t}\right\} \\
& =\int_{0}^{\infty} e^{-\lambda t} \psi_{h}^{*}(x, \lambda) \psi_{h}^{*}(y, \lambda) d \sigma_{h}^{*}(\lambda),
\end{aligned}
$$

where

$$
\psi_{h}^{*}(x, \lambda)=\frac{\sin (\sqrt{\lambda / a} x)}{\sqrt{\lambda / a} x}, \quad d \sigma_{h}^{*}(\lambda)=\frac{1}{\pi} \sqrt{\frac{\lambda}{a}} d \lambda .
$$

When $a=1 / 2,(6.5)$ is the generator of a three-dimensional Bessel process which we denote by $\widetilde{\mathcal{G}}$; that is,

$$
\widetilde{\mathcal{G}}=\frac{1}{2} \frac{d^{2}}{d x^{2}}+\frac{1}{x} \frac{d}{d x}
$$


Since $\widetilde{h}(x)=1 / x$ satisfies $\widetilde{\mathcal{G}} \tilde{h}=0$, we can consider the $h$ transform of $\widetilde{\mathcal{G}}$ with respect to $\widetilde{h}(x)=1 / x$, which we denote by $\mathcal{G}$. Then we get

$$
\mathcal{G}=\frac{1}{2} \frac{d^{2}}{d x^{2}}
$$

This is the generator of Brownian motion on $(0, \infty)$. The above observation shows that $\mathcal{G}$ is $h$ transformed to $\widetilde{\mathcal{G}}$ in terms of $h(x)=x$, and $\widetilde{\mathcal{G}}$ is $h$ transformed to $\mathcal{G}$ in terms of $\widetilde{h}(x)=1 / x$. This fact is also obtained by means of Theorem 1.4.

(3) We denote $\mathcal{G}_{s, m, k}$ with $b^{2}+c>0$ (respectively $b^{2}+c=0$ ) by $\mathcal{G}^{(1)}$ (respectively $\left.\mathcal{G}^{(2)}\right)$. Fix a $\beta \geq 0$ arbitrarily, and set $h^{(1)}(x)=e^{\lambda_{1}(\beta) x}$. Since $\mathcal{G}^{(1)} h^{(1)}=\beta h^{(1)}$, we can consider an $h$ transform of $\mathcal{G}^{(1)}$ with respect to $h^{(1)}$ which is denoted by $\widetilde{\mathcal{G}}^{(1)}$; that is,

$$
\begin{aligned}
\widetilde{\mathcal{G}}^{(1)} & =a \frac{d^{2}}{d x^{2}}+\left(b+2 a \frac{h^{(1)^{\prime}}(x)}{h^{(1)}(x)}\right) \frac{d}{d x} \\
& =a \frac{d^{2}}{d x^{2}}+\sqrt{b^{2}+4 a(c+\beta)} \frac{d}{d x} .
\end{aligned}
$$

We put $\kappa=b^{2} / 4 a+c+\beta$ and $h^{(2)}(x)=e^{\sqrt{\kappa / a} x}$. Since $\mathcal{G}^{(2)} h^{(2)}=\kappa h^{(2)}$, we can consider an $h$ transform of $\mathcal{G}^{(2)}$ with respect to $h^{(2)}$ which is denoted by $\widetilde{\mathcal{G}}^{(2)}$; that is,

$$
\begin{aligned}
\widetilde{\mathcal{G}}^{(2)} & =a \frac{d^{2}}{d x^{2}}+2 a \frac{h^{(2)^{\prime}}(x)}{h^{(2)}(x)} \frac{d}{d x} \\
& =a \frac{d^{2}}{d x^{2}}+2 a \sqrt{\kappa / a} \frac{d}{d x} \\
& =a \frac{d^{2}}{d x^{2}}+\sqrt{b^{2}+4 a(c+\beta)} \frac{d}{d x} .
\end{aligned}
$$

We find that $\mathcal{G}^{(1)} \neq \mathcal{G}^{(2)}$, but $\widetilde{\mathcal{G}}^{(1)}=\widetilde{\mathcal{G}}^{(2)}$. This shows that an $h$ transform of ODGDO is not a one-to-one correspondence.

Example 6.2. Let us consider the following ODGDO $\mathcal{G}_{(\kappa)}$ on $I=(0, \infty)$ :

$$
\mathcal{G}_{(\kappa)}=\frac{1}{2} \frac{d^{2}}{d x^{2}}+\frac{\kappa}{2 x} \frac{d}{d x}
$$

where $\kappa$ is a real number. $\mathcal{G}_{(0)}$ is the generator of Brownian motion on $(0, \infty)$, and $\mathcal{G}_{(\kappa)}$ is the generator of a $(\kappa+1)$-dimensional Bessel process for $\kappa \in \mathbb{N}$. We may set the scale function $s_{(\kappa)}$ and the speed measure $m_{(\kappa)}$ as

$$
d s_{(\kappa)}(x)=x^{-\kappa} d x, \quad d m_{(\kappa)}(x)=2 x^{\kappa} d x .
$$

The end point 0 is $\left(s_{(\kappa)}, m_{(\kappa)}, 0\right)$-exit, or -regular, or -entrance according to if $\kappa \leq-1$, or $-1<\kappa<1$, or $1 \leq \kappa$. The end point $\infty$ is always natural. The $\alpha$-Green function $G(\alpha, x, y)$ corresponding to $\mathcal{G}_{(\kappa)}$ is given by the following. We put $\mu=(1-\kappa) / 2$, and hence $\mu>0$ or $\mu \leq 0$ according to if $\kappa<1$ or $\kappa \geq 1$ :

$$
G_{(\kappa)}(\alpha, x, y)=G_{(\kappa)}(\alpha, y, x)= \begin{cases}(x y)^{\mu} I_{|\mu|}(\sqrt{2 \alpha} x) K_{|\mu|}(\sqrt{2 \alpha} y), & \text { if } \alpha>0, \\ (2 \mu)^{-1} x^{2 \mu}, & \text { if } \kappa<1, \alpha=0,\end{cases}
$$


for $0<x \leq y<\infty$, where $I_{v}$ and $K_{v}$ are the modified Bessel functions defined by

$$
\begin{gathered}
I_{v}(x)=\left(\frac{x}{2}\right)^{v} \sum_{n=0}^{\infty} \frac{(x / 2)^{2 n}}{n ! \Gamma(v+n+1)}, \quad v>-1, \\
K_{v}(x)(x)=K_{-v}(x)= \begin{cases}\frac{\pi}{2} \frac{I_{-v}(x)-I_{v}(x)}{\sin v \pi}, & v \in \mathbb{Z}, \\
\frac{(-1)^{v}}{2} \lim _{\xi \rightarrow v}\left(\frac{\partial I_{-\xi}(x)}{\partial \xi}-\frac{\partial I_{\xi}(x)}{\partial \xi}\right), & v \notin \mathbb{Z} .\end{cases}
\end{gathered}
$$

The elementary solution $p_{(\kappa)}(t, x, y)$ is given as

$$
p_{(\kappa)}(t, x, y)=\frac{1}{2 t} \exp \left\{-\frac{x^{2}+y^{2}}{2 t}\right\}(x y)^{\mu} I_{|\mu|}\left(\frac{x y}{t}\right), \quad t, x, y>0 .
$$

We note the formula (see [6, p. 200])

$$
\int_{0}^{\infty} e^{-\lambda t} J_{v}(\sqrt{\lambda} x) J_{v}(\sqrt{\lambda} y) d \lambda=\frac{1}{t} \exp \left\{-\frac{x^{2}+y^{2}}{4 t}\right\} I_{v}\left(\frac{x y}{2 t}\right),
$$

for $t, x, y>0$ and $v>-1$, where $J_{v}$ is the Bessel function defined by

$$
J_{v}(x)=\left(\frac{x}{2}\right)^{v} \sum_{n=0}^{\infty} \frac{(-1)^{n}(x / 2)^{2} n}{n ! \Gamma(v+n+1)} .
$$

By means of (6.9) and (6.10), we get the representation

$$
p_{(\kappa)}(t, x, y)=\int_{0}^{\infty} e^{-\lambda t} \psi_{(\kappa)}(x, \lambda) \psi_{(\kappa)}(y, \lambda) \sigma_{(\kappa)}(\lambda) d \lambda,
$$

where

$$
\psi_{(\kappa)}(x, \lambda)=C_{(\kappa)}(\lambda) x^{\mu} J_{|\mu|}(\sqrt{2 \lambda} x), \quad \sigma_{(\kappa)}(\lambda)=C_{(\kappa)}(\lambda)^{-2},
$$

and $C_{(\kappa)}(\lambda)$ is a positive number satisfying

$$
\begin{aligned}
\lim _{x \rightarrow 0} \psi_{(\kappa)}(x, \lambda)=1 & \text { if } \kappa \geq 1, \\
\lim _{x \rightarrow 0} D_{s_{(\kappa)}} \psi_{(\kappa)}(x, \lambda)=1 & \text { if } \kappa<1,
\end{aligned}
$$

so that,

$$
C_{(\kappa)}(\lambda)= \begin{cases}2^{-1}(\lambda / 2)^{-\mu / 2} \Gamma(\mu), & \text { if } \kappa<1 \\ (\lambda / 2)^{-|\mu| / 2} \Gamma(1+|\mu|), & \text { if } \kappa \geq 1\end{cases}
$$

For $\beta>0$, we set

$$
h_{(\kappa)}(x)=(\sqrt{2 \beta} x)^{\mu} K_{|\mu|}(\sqrt{2 \beta} x) .
$$

The function $h_{(\kappa)}$ is positive and satisfies $\mathcal{G}^{(\kappa)} h_{(\kappa)}=\beta h_{(\kappa)}$. We denote by $\widetilde{\mathcal{G}}_{(\kappa)}$ the $h$ transform of $\mathcal{G}_{(\kappa)}$ with respect to $h_{(\kappa)}$; that is,

$$
\begin{aligned}
\tilde{\mathcal{G}}_{(\kappa)} & =\frac{1}{2} \frac{d^{2}}{d x^{2}}+\left(\frac{\kappa}{2 x}+\frac{h_{(\kappa)}^{\prime}(x)}{h_{(\kappa)}(x)}\right) \frac{d}{d x} \\
& =\frac{1}{2} \frac{d^{2}}{d x^{2}}+\left\{\frac{1}{2 x}+\sqrt{2 \beta} \frac{K_{|\mu|}^{\prime}(\sqrt{2 \beta} x)}{K_{|\mu|}(\sqrt{2 \beta} x)}\right\} \frac{d}{d x} .
\end{aligned}
$$


Since $h_{(\kappa)}(0) \in(0, \infty)$ (respectively $\left.=\infty\right)$ if $\kappa \in(-\infty, 1)$ (respectively $\in[1, \infty)$ ), and $\left|\widetilde{m}_{(\kappa)}(0)\right| \in[0, \infty)$ (respectively $=\infty$ ) if $\kappa \in[1,3$ ) (respectively $\in[3, \infty)$ ), the end point 0 is $\left.\widetilde{s}_{(\kappa)}, \widetilde{m}_{(\kappa)}, 0\right)$-regular or -exit according to if $\kappa<3$ or $\kappa \geq 3$, where

$$
d \widetilde{s}_{(\kappa)}(x)=h_{(\kappa)}(x)^{-2} x^{-\kappa} d x, \quad d \widetilde{m}_{(\kappa)}(x)=2 h_{(\kappa)}(x)^{2} x^{\kappa} d x .
$$

We denote by $\tilde{p}_{(\kappa)}(t, x, y)$ the elementary solution of equation (1.1) with $\mathcal{G}_{s, m, k}$ replaced by $\widetilde{\mathcal{G}}_{(\kappa)}$. Then we get

$$
\begin{aligned}
\tilde{p}_{(\kappa)}(t, x, y) & =e^{-\beta t} p_{(\kappa)}(t, x, y) / h_{(\kappa)}(x) h_{(\kappa)}(y) \\
& =\frac{1}{2(2 \beta)^{\mu} t} \exp \left\{-\beta t-\frac{x^{2}+y^{2}}{2 t}\right\} I_{|\mu|}\left(\frac{x y}{t}\right) / K_{|\mu|}(\sqrt{2 \beta} x) K_{|\mu|}(\sqrt{2 \beta} y) \\
& =\int_{\beta}^{\infty} e^{-\lambda t} \widetilde{\psi}_{(\kappa)}(x, \lambda) \widetilde{\psi}_{(\kappa)}(x, \lambda) \widetilde{\sigma}_{(\kappa)}(\lambda) d \lambda
\end{aligned}
$$

where $\widetilde{\psi}_{(\kappa)}(x, \lambda)$ and $\widetilde{\sigma}_{(\kappa)}(\lambda)$ are obtained by means of (5.13), (5.14), (5.17) and (5.18).

We now take a $\kappa_{1} \in(-\infty, 1)$ and put $\kappa_{2}=2-\kappa_{1} \in(1, \infty)$. Then $\left|\left(1-\kappa_{1}\right) / 2\right|=$ $\left|\left(1-\kappa_{2}\right) / 2\right|$. We thus find that $\mathcal{G}_{\left(\kappa_{1}\right)} \neq \mathcal{G}_{\left(\kappa_{2}\right)}$, but $\widetilde{\mathcal{G}}_{\left(\kappa_{1}\right)}=\widetilde{\mathcal{G}}_{\left(\kappa_{2}\right)}$. This also shows that an $h$ transform of ODGDO is not a one-to-one correspondence.

Example 6.3. Finally we consider the following ODGDO $\mathcal{G}_{o}$ on $(0, \infty)$ :

$$
\mathcal{G}_{o}=\frac{1}{2} \frac{d^{2}}{d x^{2}}-c x^{-2}
$$

where $c$ is a positive number. We may set

$$
d s_{o}(x)=d x, \quad d m_{o}(x)=2 d x, \quad d k_{o}(x)=2 c x^{-2} d x .
$$

We note that both of the end points 0 and $\infty$ are $\left(s_{o}, m_{o}, k_{o}\right)$-natural.

Let $p_{o}(t, x, y)$ be the elementary solution of equation $(1.1)$ with $\mathcal{G}_{s, m, k}$ replaced by $\mathcal{G}_{o}$. By using Proposition 3.3, we show that $p_{o}(t, x, y)$ is given by (6.19) below.

For $\beta>0$, we set

$$
h_{o}(x)=\sqrt{x} K_{v}(\sqrt{2 \beta} x),
$$

where $v=\sqrt{2 c+1 / 4}(>1 / 2)$. The function $h_{o}$ is positive and satisfies $\mathcal{G}_{o} h_{o}=\beta h_{o}$. We denote by $\widetilde{\mathcal{G}}_{o}$ the $h$ transform of $\mathcal{G}$ with respect to $h_{o}$. Then we find

$$
\begin{aligned}
\widetilde{\mathcal{G}}_{o} & =\frac{1}{2} \frac{d^{2}}{d x^{2}}+\frac{h_{o}^{\prime}(x)}{h_{o}(x)} \frac{d}{d x} \\
& =\frac{1}{2} \frac{d^{2}}{d x^{2}}+\left\{\frac{1}{2 x}+\sqrt{2 \beta} \frac{K_{v}^{\prime}(\sqrt{2 \beta} x)}{K_{v}(\sqrt{2 \beta} x)}\right\} \frac{d}{d x} .
\end{aligned}
$$

This shows that $\widetilde{\mathcal{G}}_{o}$ coincides with $\widetilde{\mathcal{G}}_{(\kappa)}$ with $v=|\mu|=|(1-\kappa) / 2|$ in the preceding example. It should be noted that we take the scale $\widetilde{s}_{o}$ (respectively $\widetilde{s}_{(\kappa)}$ ) and the speed measure $\tilde{m}_{o}$ (respectively $\tilde{m}_{(\kappa)}$ ) corresponding to $\widetilde{\mathcal{G}}_{o}$ (respectively $\widetilde{\mathcal{G}}_{(\kappa)}$ ), where

$$
d \widetilde{s}_{o}(x)=h_{o}(x)^{-2} d x, \quad d \widetilde{m}_{o}(x)=2 h_{o}(x)^{2} d x .
$$


Let $\tilde{p}_{o}(t, x, y)$ be the elementary solution of equation (1.1) with $\mathcal{G}_{s, m, k}$ replaced by $\widetilde{\mathcal{G}}_{o}$. Then we have

$$
\tilde{p}_{o}(t, x, y)=e^{-\beta t} p_{o}(t, x, y) / h_{o}(x) h_{o}(y) .
$$

Let $v=|(1-\kappa) / 2|$. Since $\widetilde{\mathcal{G}}_{o}$ coincides with $\widetilde{\mathcal{G}}_{(\kappa)}$, noting (6.16) and (6.13), we see that

$$
\tilde{p}_{o}(t, x, y) h_{o}(y)^{2}=\tilde{p}_{(\kappa)}(t, x, y) h_{(\kappa)}(y)^{2} y^{\kappa} .
$$

By using (6.9), (6.11), (6.14), (6.17), and (6.18), we obtain

$$
\begin{aligned}
p_{o}(t, x, y) & =p_{(\kappa)}(t, x, y) \frac{h_{(\kappa)}(y)}{h_{(\kappa)}(x)} \frac{h_{o}(x)}{h_{o}(y)} y^{\kappa} \\
& =\frac{1}{2 t} \exp \left\{-\frac{x^{2}+y^{2}}{2 t}\right\}(x y)^{1 / 2} I_{\nu}\left(\frac{x y}{t}\right) .
\end{aligned}
$$

Acknowledgement. The second author was partially supported by Grant-in-Aid for Scientific Research (C) 22540132.

\title{
REFERENCES
}

[1] W. Feller. The parabolic differential equations and the associated semi-groups of transformations. Ann. of Math. 55 (1952), 468-519.

[2] M. Fukushima, Y. Oshima and M. Takeda. Dirichlet Forms and Symmetric Markov Processes. Walter de Gruyter, Berlin, 1994.

[3] K. Itô and H. P. McKean, Jr. Diffusion Processes and their Sample Paths. Springer, New York, 1974.

[4] M. Maeno. One-dimensional $h$-path generalized diffusion processes. Ann. Reports of Graduate School of Humanities and Sciences, Nara Women's University 21 (2006), 167-185.

[5] H. P. McKean, Jr. Elementary solutions for certain parabolic differential equations. Trans. Amer. Math. Soc. 82 (1956), 519-548.

[6] S. Moriguchi, K. Udagawa and S. Hitotsumatsu. Sugaku Kosiki (in Japanese). Iwanami, 1960.

[7] T. Takemura. State of boundaries for harmonic transforms of one-dimensional generalized diffusion processes. Ann. Reports of Graduate School of Humanities and Sciences, Nara Women's University 25 (2010), 285-294.

[8] M. Tomisaki. Intrinsic ultracontractivity and small perturbation for one-dimensional generalized diffusion operators. J. Funct. Anal. 251 (2007), 289-324.

\section{Tomoko Takemura \\ Department of Mathematics \\ Nara Women's University}

Kita-Uoya Nishimachi, Nara, 630-8506

Japan

(E-mail:sm18031@cc.nara-wu.ac.jp)

\author{
Matsuyo Tomisaki \\ Department of Mathematics \\ Nara Women's University \\ Kita-Uoya Nishimachi, Nara, 630-8506 \\ Japan \\ (E-mail: tomisaki@cc.nara-wu.ac.jp)
}

